\title{
Enfermagem e o uso de tecnologias nos serviços de terapia antineoplásica brasileiro
}

RESUMO | Objetivo: descrever as principais tecnologias utilizadas pela equipe de enfermagem nos serviços de terapia antineoplásica do Brasil. Método: pesquisa de revisão narrativa da literatura, com busca nas bases de dados LILACS (Literatura Latino-Americana e do Caribe em Ciências da Saúde) e MEDLINE (Medical Literature Analysis and Retrieval Sistem On-line) via Biblioteca Virtual de Saúde (BVS). Os critérios de inclusão foram estudos em português publicados em periódico nacional, com recorte atemporal e abordagem sobre o uso de tecnologias em serviços de terapia antineoplásicas. Resultados e Discussão: os estudos encontrados na literatura foram publicados nos anos de 2004, 2007, 2008, 2010, 2011, 2013, 2014, 2015 e 2016. As Tecnologias utilizadas pela enfermagem foram do tipo leves, leve-duras e duras, materializadas em vídeo educativo, brinquedo terapêutico, software, dispositivo para infusão contínua, processo de enfermagem, música, comunicação, acolhimento e relação interpessoal. Foi possível observar muitos benefícios advindos do uso das tecnologias assistenciais. Conclusão: a enfermagem utiliza diversos tipos de tecnologias na prestação da assistência ao paciente submetido à terapia antineoplásica, as mesmas trazem grandes benéficos ao paciente e a profissão que caminha lado a lado dos avanços tecnológicos da humanidade.

Palavras-chaves: Tecnologia Biomédica; Tecnologia em Saúde; Antineoplásicos; Profissionais de Enfermagem.

ABSTRACT | Objective: to describe the main technologies used by the nursing staff in antineoplastic therapy services in Brazil. Method: research of narrative literature review, searching LILACS (Latin American and Caribbean Literature in Health Sciences) and MEDLINE (Medical Literature Analysis and Retrieval System Online) databases via Virtual Health Library (VHL). The inclusion criteria were studies in Portuguese published in a national journal, with a timeless cut and approach on the use of technologies in antineoplastic therapy services. Results and Discussion: the studies found in the literature were published in the years 2004, 2007, 2008, 2010, 2011, 2013, 2014, 2015 and 2016. The technologies used by nursing were light, light-hard and hard, materialized in educational video, therapeutic toy, software, device for continuous infusion, nursing process, music, communication, welcoming and interpersonal relationship. It was possible to observe many benefits from the use of assistive technologies. Conclusion: nursing uses several types of technologies in providing care to patients undergoing antineoplastic therapy, they bring great benefits to the patient and the profession that goes hand in hand with the technological advances of humanity.

Keywords: Biomedical Technology; Health Technology; Antineoplastics; Nursing Professionals.

RESUMEN | Objetivo: describir las principales tecnologías utilizadas por el personal de enfermería en los servicios de terapia antineoplásica en Brasil. Método: investigación de revisión de literatura narrativa, búsqueda en bases de datos LILACS (Literatura Latinoamericana y del Caribe en Ciencias de la Salud) y MEDLINE (Sistema de Análisis y Recuperación de Literatura Médica en línea) a través de la Biblioteca Virtual en Salud (BVS). Los criterios de inclusión fueron estudios en portugués publicados en una revista nacional, con un corte y un enfoque intemporales sobre el uso de tecnologías en los servicios de terapia antineoplásica. Resultados y discusión: los estudios encontrados en la literatura se publicaron en los años 2004, 2007, 2008, 2010, 2011, 2013, 2014, 2015 y 2016. Las tecnologías utilizadas por la enfermería fueron ligeras, duras y duras, materializadas en video educativo, juguete terapéutico, software, dispositivo para infusión continua, proceso de enfermería, música, comunicación, bienvenida y relación interpersonal. Fue posible observar muchos beneficios del uso de tecnologías de asistencia. Conclusión: la enfermería utiliza varios tipos de tecnologías para brindar atención a los pacientes que reciben terapia antineoplásica, aportan grandes beneficios para el paciente y la profesión que van de la mano con los avances tecnológicos de la humanidad.

Palavras claves: Tecnología Biomédica; Tecnología de Salud; Antineoplásticos; Profesionales de Enfermería.

\section{Evandro Bernardino Mendes De Melo}

Enfermeiro, doutorando em enfermagem pela Universidade Federal de Minas Gerais. https://orcid.org/0000-0002-1772-3083

\section{Marcelo Costa Vicente}

Mestre em saúde coletiva, Faculdades Integradas São Pedro; https://orcid. org/0000-0002-5518-2900

\section{Marcelo dos Santos Pinto}

Enfermeiro, especialista em docência do ensino superior, Escola Politecnica do Brasil; https://orcid.org/0000-0002-9605-7874

\section{Bárbara Heloisa Soares Heringer Xavier}

Enfermeira com licenciatura em biologia,Escola Politecnica do Brasil. https://orcid.org/0000-0001-8792-8909

\section{Claudia Curbani Vieira Manola}

Enfermeira, mestre em administração, Centro Universitário Salesiano de Vitória; https://orcid.org/0000-0002-0925-4432

\section{Livia Perasol}

Enfermeira, doutora em educação, Centro Universitário Salesiano de Vitória; https:// orcid.org/0000-0001-8228-6468

Recebido em: 07/06/2020

Aprovado em: 07/06/2020
INTRODUÇÃO

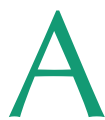
s tecnologias podem ser definidas como produtos que envolvem um conjunto de instrumentos, métodos e técnicas que visam a resolução de problemas do dia a dia das pessoas ${ }^{(1)}$. É também constituída pela aplicação prática do conhecimento científico em diversas áreas da saúde ${ }^{(2)}$. Para autores ${ }^{(3: 98)}$, a palavra tecnologia significa etimologicamente o "saber fazer", ou seja, a razão do saber fazer em saúde. Autor ${ }^{(4)}$ classifica as tecnologias em 
três categorias: "duras" quando envolve equipamentos, "leve-duras", próprias dos saberes estruturados, normas, protocolos e conhecimentos, e leve, das relações.

Nos diversos processos assistências que envolvem a enfermagem oncológica, as tecnologias estão relacionadas diretamente com o cuidado, elas envolvem os pacientes e os profissionais de saúde em diversos níveis de atenção, principalmente nos serviços de terapia antineoplásica, através de investigações, aplicações de teorias e da experiência cotidiana contribuindo com o desenvolvimento técnico científico, com ações sistematizadas, processuais e instrumentais para prestação de uma assistência qualificada ao ser humano ${ }^{(5)}$.

Sendo assim, a tecnologia está presente nas atividades inerentes aos profissionais de enfermagem, atualmente sabe-se que a incidência do câncer vem crescendo a cada dia no Brasil e no mundo. Estima-se que, em 2020, o número de casos anuais seja da ordem de 15 milhões. A quimioterapia (QT) constitui uma das modalidades de maior opção para o tratamento do câncer. De acordo com sua finalidade, a QT pode ser classificada em: curativa, que objetiva a erradicação de evidências das células cancerosas; paliativa, que visa minimizar sintomas decorrentes da proliferação tumoral aumentando a sobrevida. Pode ainda ser classificada de acordo com o período do tratamento em que é realizada, podendo ser adjuvante, ou seja, realizada depois de um tratamento principal, como por exemplo, a cirurgia e; neoadjuvante, quando é realizada previamente ao tratamento principal ${ }^{(6,7)}$.

Nesse contexto, a Resolução do Conselho Federal de Enfermagem (COFEN) n. ${ }^{\circ}$ 210/1998 apresenta importantes contribuições ao regulamentar a atuação do enfermeiro nos serviços de terapia antineoplásica, afirmando que é dever desse profissional "atuar ativamente utilizando recursos terapêuticos de enfermagem na prevenção, tratamento e minimização dos efeitos colaterais em clientes subme-

\section{6}

Estima-se que, em 2020, o número de casos anuais seja da ordem de 15 milhões. A quimioterapia (QT) constitui uma das modalidades de maior opção para o tratamento do câncer. De acordo com sua finalidade, a QT pode ser classificada em: curativa, que objetiva a erradicação de evidências das células cancerosas; paliativa, que visa minimizar sintomas decorrentes da proliferação tumoral aumentando a sobrevida. tidos ao tratamento antineoplásico"(8). De fato, o uso de tecnologias assistenciais é primordial para a continuidade do cuidado junto ao paciente submetido à quimioterapia, sendo ao mesmo tempo processo e produto melhorando a qualidade de vida durante o tratamento ${ }^{(9)}$.

Diante do exposto, surgiu a seguinte pergunta: Quais as evidências na literatura brasileira sobre o uso de tecnologias na assistência de enfermagem em paciente submetidos à terapia antineoplásica? Nessa perspectiva, o presente trabalho teve como objetivo descrever as principais tecnologias utilizadas pela equipe enfermagem nos serviços de terapia antineoplásica do Brasil.

\section{METODOLOGIA}

Pesquisa de revisão narrativa da literatura, este método permite a síntese de múltiplos estudos publicados e possibilita conclusões gerais acerca de uma particular área de estudo(10).

A pesquisa foi construída a partir das seguintes etapas: desenvolvimento da questão norteadora, busca dos estudos nas bases de dados, extração dos dados dos estudos avaliados, análise e síntese dos resultados, conforme proposta por autores ${ }^{(11)}$.

Inicialmente para direcionar este estudo foi elaborada a seguinte questão norteadora: "Quais as evidências na literatura brasileira sobre o uso de tecnologias na assistência de enfermagem em paciente submetidos a terapia antineoplásica?". A coleta de dados foi realizada eletronicamente através da utilização da internet no mês de outubro de 2017, através das seguintes bases de dados LILACS (Literatura Latino-Americana e do Caribe em Ciências da Saúde) e MEDLINE (Medical Literature Analysis and Retrieval Sistem On-line) via Biblioteca Virtual de Saúde (BVS). Os descritores utilizados foram: "tecnologia biomédica", "tecnologia em saú$\mathrm{de}^{\prime}$, "antineoplásicos" e "profissionais de enfermagem", foram combinados 
de diferentes formas para garantir uma busca mais ampla da literatura conforme Tabela 1.

Os critérios de inclusão para a pré-seleção dos estudos foram: estudos em português publicados em periódico nacional com recorte atemporal e com abordagem sobre o uso de tecnologias em serviços de terapia antineoplásica. Foram excluídos os estudos que não responderam ao objetivo da pesquisa. A seleção dos estudos foi realizada por meio da leitura seqüencialmente dos títulos, resumos e por último leitura na íntegra. A busca inicial permitiu a obtenção de um total de 4.912 artigos que, após a leitura dos títulos, dos resumos e posterior análise na íntegra dos textos, foram excluídos 4.897 e selecionados de 15 artigos que apresentavam relação com o tema em questão.

\section{RESULTADOS E DISCUSSÃO}

Considerando a análise e síntese dos resultados da presente pesquisa, foi possível identificar a utilização de tecnologias do tipo leve, leve dura e dura na prática dos profissionais de enfermagem aplicadas ao paciente em tratamento quimioterápico. As tecnologias encontradas foram materializadas através de vídeo educativo, brinquedo terapêutico, software, dispositi-

Tabela 1. Cruzamento dos descritores e seleção dos artigos do estudo. Vitória, ES, Brasil, 2017

\begin{tabular}{|c|c|c|c|}
\hline \multirow{7}{*}{$\begin{array}{l}\text { BIBLIOTECA } \\
\text { VIRTUAL DE } \\
\text { SAÚDE } \\
\text { (BVS) }\end{array}$} & CRUZAMENTO ENTRE OS DESCRITORES & $\begin{array}{l}\text { NÚMERO } \\
\text { DE ARTIGOS }\end{array}$ & TOTAL \\
\hline & "Tecnologia biomédica" x "Antineoplásicos". & 103 & \multirow[b]{2}{*}{ 4.912 ARTIGOS } \\
\hline & $\begin{array}{l}\text { "Tecnologia biomédica" x "Tecnologia em } \\
\text { Saúde }\end{array}$ & 551 & \\
\hline & $\begin{array}{c}\text { "Tecnologia biomédica" x "Profissionais de } \\
\text { Enfermagem" }\end{array}$ & 459 & \multirow{4}{*}{$\begin{array}{c}\text { EXCLUIDOS } \\
4.897 \\
\text { ARTIGOS }\end{array}$} \\
\hline & "Tecnologia em saúde" x "Antineoplásicos" & 2.526 & \\
\hline & $\begin{array}{c}\text { "Tecnologia em saúde" x "Profissionais de } \\
\text { Enfermagem" }\end{array}$ & 784 & \\
\hline & $\begin{array}{c}\text { "Antineoplásicos" x "Profissionais de } \\
\text { Enfermagem" }\end{array}$ & 489 & \\
\hline
\end{tabular}

Gráfico 1. Classificação das tecnologias apresentadas por ano de publicação. Vitória, ES, Brasil, 2017

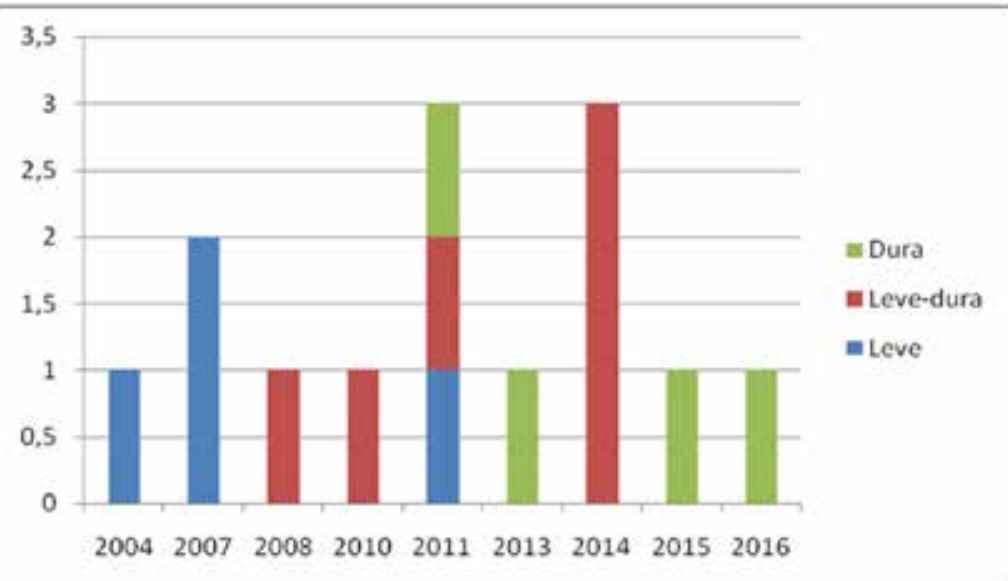

vo para infusão contínua, processo de enfermagem, músicoterapia, comunicação, acolhimento e relação interpessoal. De acordo com os critérios de Merhy ${ }^{(4)}$ e com base nos achados da presente pesquisa, foi elaborado um gráfico com as principais classificações das tecnologias por ano de publicação conforme mostra abaixo:

Os estudos foram publicados entre nos anos de 2004, 2007, 2008, 2010, 2011, 2013, 2014, 2015 e 2016, sendo possível identificar a utilização de tecnologias duras, leve-duras e leves nos serviços de terapia antineoplásica por parte da equipe de enfermagem.

$M^{2}{ }^{(4)}$ foi pioneiro ao classificar as tecnologias como leves, leves-dura ou duras, sendo: a tecnologia dura representada pelo material concreto como equipamentos tecnológicos, mobiliário tipo permanente ou de consumo, já a tecnologia leve-dura, representada pelos saberes estruturados e disciplinas que operam em saúde, a exemplo da clínica médica, odontológica, epidemiológica, teorias, modelos de cuidado, processo de enfermagem entre outras, e a tecnologia leve que se expressa como o processo de produção da comunicação, das relações, de vínculos que conduzem ao encontro do usuário com necessidades de ações de saúde como vínculo, gestão de serviços e acolhimento.

As três classificações delineadas estão estreitamente interligadas e presentes no agir da enfermagem, embora nem sempre de modo transparente descrito como tecnologia de enfermagem. Tanto para área da saúde como para enfermagem, a produção acelerada da ciência e da tecnologia é essencial para as inovações; influenciam diretamente na organização do serviço, no modo de cuidar, inovando e fundamentando a prática assistencial ${ }^{(4,12)}$.

Embora essas três categorias se inter-relacionem, o ser humano necessita, em especial, das tecnologias de relações, definidas como 'leves'. Elas são capazes de propiciar o acolhimento necessário para que cliente e profissional de saúde possam se beneficiar deste momento, considerando a complexidade do ser 
humano o sujeito é contextualizado, estando seu estado de saúde dependente das condições ambientais, biológicas, psicológicas, do seu estilo de vida e das instituições em que se opera o cuidado. A conjunção desses fatores interfere nas tecnologias incorporadas à saúde $\mathrm{e}^{(4,13)}$. Considerando as principais tecnologias do tipo leves encontradas neste estudo, a Tabela 2 evidencia as tecnologias leves utilizadas pelos profissionais de enfermagem na assistência direcionada ao paciente oncológico em serviços de terapia antineoplásica.

$\mathrm{Na}$ presente pesquisa foi possível identificar as tecnologias do tipo leves materializadas em: relacionamento terapêutico, comunicação verbal e não verbal, exercício da fé e esperitualidade e acolhimento. Os estudos foram publicados nos anos de 2005, 2007, 2011 e 2014. As tecnologias leves utilizam atributos que são próprios da relação humana, fundamentais na construção de vínculo entre os profissionais de enfermagem e o cliente no espaço do cuidado prestado ao paciente oncológico ${ }^{(14)}$.

A pesquisa mostrou que uma das tecnologias de grande relevância foi a interação terapêutica entre o profissional de enfermagem e o paciente. Uma pesquisa ${ }^{(15)}$ avaliou os benefícios da interação terapêutica entre os profissionais de enfermagem e o paciente oncológico, chegando à conclusão que tal prática favorece o tratamento proposto aos pacientes, ajudan- do-os a se adaptarem melhor a quimioterapia, além de transmitir-lhes confiança e segurança, diminuindo o medo e a ansiedade. Autores ${ }^{(14)}$ citam que a interação terapêutica é fundamental para um cuidado humanizado na demonstração de respeito por parte do enfermeiro, sendo imprensidivel a este profissional o desenvolvimento de uma comunicação adequada e efetiva, utilizando-se de procedimentos técnicos, escuta e atenção adequada ao paciente oncológico.

Contribuindo com os achados supracitados, um estudo(16) utilizou a comunicação terapêutica em 39 pacientes em cuidados paliativos submetidos à terapia antineoplásica e chegou à conclusão de que a comunicação verbal e não verbal

\section{Tabela 2. Análise dos Artigos selecionados segundo classificação das Tecnologias - "Leve". Vitória, ES, Brasil, 2017}

\begin{tabular}{|c|c|c|c|c|c|}
\hline Referências & Nome do Artigo & $\begin{array}{c}\text { Característica } \\
\text { da Amostra/ } \\
\text { População }\end{array}$ & $\begin{array}{l}\text { Tecnologia } \\
\text { estudada }\end{array}$ & Papel da Enfermagem & Resultados / Expectativas \\
\hline $\begin{array}{l}\text { Marques, Laranja e Silva. } \\
\text { Revista da UFPE Enferma- } \\
\text { gem Brasil, } 2005\end{array}$ & $\begin{array}{l}\text { Os clientes e } \\
\text { os enfermeiros: } \\
\text { construção de } \\
\text { uma relação }\end{array}$ & $\begin{array}{l}\text { Grupo focal na } \\
\text { relação entre } \\
5 \text { enfermeiros } \\
\text { e } 10 \text { pacientes } \\
\text { em tratamento } \\
\text { oncológico. }\end{array}$ & $\begin{array}{l}\text { Relacionamento } \\
\text { terapêutico na } \\
\text { interação com o } \\
\text { paciente onco- } \\
\text { lógico }\end{array}$ & $\begin{array}{l}\text { Utilizar ferramentas que } \\
\text { favoreçam a interação } \\
\text { entre os profissionais e } \\
\text { pacientes em tratamen- } \\
\text { to quimioterápico }\end{array}$ & $\begin{array}{l}\text { A enfermagem deve utilizar } \\
\text { ferramentas alternativas para } \\
\text { gerir o relacionamento tera- } \\
\text { pêutico, além do conceito de } \\
\text { "educação terapêutica". }\end{array}$ \\
\hline
\end{tabular}

\section{Entrevista com}

39 pacientes

Araújo e Silva, Revista da Escola de Enfermagem da USP Brasil, 2007

\section{A comunicação com o paciente} em cuidados paliativos: valorizando a alegria e 0 otimismo

\section{em tratamento}

oncológico com

comunicação

verbal preserva-

da, submetidos

à quimioterapia

paliativa

\begin{tabular}{|c|c|c|c|c|c|}
\hline $\begin{array}{l}\text { Peres, et al.,Revista de } \\
\text { Psiquiatria Clinica Brasil, } \\
2011\end{array}$ & $\begin{array}{c}\text { A importância } \\
\text { da integração da } \\
\text { espiritualidade e } \\
\text { da religiosidade } \\
\text { no manejo da dor } \\
\text { e dos cuidados } \\
\text { paliativos }\end{array}$ & $\begin{array}{l}\text { Entrevista com } \\
15 \text { pacientes } \\
\text { em tratamento } \\
\text { paliativo que } \\
\text { apresentavam } \\
\text { dor crônica. }\end{array}$ & $\begin{array}{l}\text { Interação } \\
\text { espiritual (fé, } \\
\text { espiritualidade e } \\
\text { religiosidade) do } \\
\text { profissional de } \\
\text { enfermagem no } \\
\text { momento da dor } \\
\text { oncológica }\end{array}$ & $\begin{array}{l}\text { Praticar a fé e espiritu- } \\
\text { alidade no tratamento } \\
\text { dos pacientes paliativos } \\
\text { que experimentam a } \\
\text { dor crônica }\end{array}$ & $\begin{array}{l}\text { Espiritualidade, fé e a reli- } \\
\text { giosidade são importantes } \\
\text { estratégias de enfrentamento } \\
\text { no tratamento do câncer }\end{array}$ \\
\hline
\end{tabular}

A comunicação (verbal e não-verbal) interpessoal é um importante atributo ao profissional de enfermagem comunicação

terapêutica
Conhecer as principais técnicas de comunicação terapêutica utilizadas com o paciente oncológico
Espiritualidade, fé e a relisão importantes tratégias de enfrentamento enfermagem no momento da dor
Acolhimento interação e diágo na primeira consulta de nfermagem

\section{.}


são importantes atributos utilizados pelos enfermeiros no exercício do cuidado, abrindo canais de comunicação promovendo alegria e otimismo aos parcientes durante o tratamento quimioterápico.

Autores $^{(17)}$ corroboram dizendo que o enfermeiro deve possuir conhecimentos e habilidades operacionalizadas no saber escutar, falar quando necessário, dar abertura para realização de perguntas, ser honesto, mostrar respeito, dispensar tempo suficiente para a conversa e mostrar interesse. Citam ainda, que a comunicação não é simplesmente uma troca de mensagens entre o enfermeiro e o paciente, mas é uma ação que deve ser planejada e individualizada, não sendo realizada somente por impulsos e de forma intuitiva.

O exercício da espiritualidade, a fé, e a religiosidade foram identificadas como tecnologias leves vivenciadas na práti- ca do enfermeiro. Estudo(18) ao avaliar 15 pacientes com dor crônica submetidos a quimioterapia paliativa, identificou que a fé, a espiritualidade e a religiosidade foram estratégias de enfrentamento utilizadas por parte dos pacientes durante $\mathrm{o}$ tratamento, promovendo confiança e emponderamento no que concerne ao tratamento proposto. Tais achados corroboram com o estudo ${ }^{(19)}$ que ao avaliar a espiritualidade, religiosidade e crenças pessoais de adolescentes com câncer, identificou que tais atributos podem emergir como componentes geradores de esperança e busca pelo sentido da vida, além de serem essenciais para o conforto e enfrentamento do estresse gerado pelo adoecimento. Citam ainda que esses atributos podem trazer os seguintes benefícios: aumento dos sentimentos de força, tranquilidade e confiança; promoção do autocuidado au- xilio a recuperação e o restabelecimento continuo, promovendo o apoio espiritual, emocional, e inclusive financeiro, contribuindo para os sentimentos de pertencimento a um grupo social; dando significado à vida, aos amigos e à família.

As tecnologias leve-duras também foram encontratas durante a análise e síntese dos estudos encontrados, foi possível identificar sete estudos publicados nos anos de 2008 (1), 2010 (1), 2011(1) e 2014 (4). Os tipos de tecnologias utilizadas foram: processo de enfermagem, cartilha informativa, instrumentos de coleta de dados, musicoterapia e análise da qualidade de vida dos pacientes submetidos à terapia antineoplásica. A Tabela 3 evidencia as principais publicações que utilizaram tecnologias do tipo "leve-dura" na assistência prestada ao paciente oncológico em serviços de terapia antineoplásica.

\section{Tabela 3. Análise dos Artigos selecionados segundo classificação das Tecnologias - "Leve-dura". Vitória, ES, Brasil, 2017}

$\begin{array}{lc}\text { Referências } & \text { Nome do Artigo } \\ \text { Anjos et al. Revista } & \begin{array}{c}\text { Sistematização da assis- } \\ \text { tência de enfermagem ao }\end{array} \\ \text { da Escola de } & \text { paciente em tratamento } \\ \text { Enfermagem da USP } & \text { quimioterápico: relato de } \\ \text { Brasil, 2011 } & \text { experiência }\end{array}$

Salles e Castro, Revista da Escola de Enfermagem da USP Brasil, 2010

Validação de material
nformativo a pacientes em
tratamento quimioterápico
e aos seus familiares

Pesquisa com 23 cuidadores principais de crianças em tratamento quimioterápico

\section{Característica da Amostra/População}

Estudantes de enfermagem e pacientes em tratamento oncológico

\section{Tecnologia estudada}

Processo de Enfermagem

\section{Papel da Enferma- gem}

Contribuir para a divulgação e desenvolvimento do processo de enfermagem
Resultados / Expectativas

0 Processo de Enfermagem foi relevante na prestação da assistência individual do paciente.

\begin{tabular}{|c|c|}
\hline $\begin{array}{l}\text { Santos et al.Revista } \\
\text { do Conselho Federal } \\
\text { de Enfermagem } \\
\text { Brasil, } 2014\end{array}$ & $\begin{array}{l}\text { Processo de enfermagem } \\
\text { aplicado ao paciente } \\
\text { oncológico }\end{array}$ \\
\hline
\end{tabular}

Silva, Marcon e Sales, Revista Brasileira de Enfermagem Brasil, 2014
Percepções de familiares de pessoas portadoras de câncer sobre encontros musicais durante o tratamento antineoplásico
Comunicação efetiva, promovendo informação adequada e atendendo as necessidades dos pacientes.
Esclarecimento de informações seguras sobre o câncer e o tratamento de quimioterapia sobre cânce e quimioterapia
12 enfermeiros que trabalham em ambulatórios de tratamento para oncologia e 20 pacientes em tratamento quimioterápico.
Elaboração e implantação de instrumento de coleta de dados em enfermagem
Emponderamento do Aumento da visibilidade do processo de enfer- enfermeiro e da qualidade da magem assistência prestada
Encontros musicais com 5 familiares
Utilização da musicoterapia
Utilizar a musicoterapia como recurso terapêutico alternativo
Promover a abertura do ser para o diálogo e o vínculo entre enfermeiro, cliente e família 


\begin{tabular}{|c|c|c|c|c|c|}
\hline $\begin{array}{l}\text { Salvadori, Lamas e } \\
\text { Zanon, Escola Anna } \\
\text { Nery Revista de } \\
\text { Enfermagem Brasil, } \\
2008\end{array}$ & $\begin{array}{l}\text { Desenvolvimento de } \\
\text { instrumento de coleta de } \\
\text { dados de enfermagem para } \\
\text { pacientes com câncer de } \\
\text { pulmão em quimioterapia } \\
\text { ambulatorial }\end{array}$ & $\begin{array}{l}\text { A população foi com- } \\
\text { posta por } 20 \text { adultos } \\
\text { de ambos os sexos, } \\
\text { portadores de câncer } \\
\text { de pulmão }\end{array}$ & $\begin{array}{l}\text { Instrumen- } \\
\text { tos de coleta } \\
\text { de dados }\end{array}$ & $\begin{array}{l}\text { Utilizar o instrumento } \\
\text { de coleta de dados } \\
\text { como parte integran- } \\
\text { te do processo de } \\
\text { enfermagem }\end{array}$ & $\begin{array}{c}\text { Teste de confiabilidade } \\
\text { mostrou-se eficaz para } \\
\text { conhecer o paciente, o tipo } \\
\text { de tratamento e os requisitos } \\
\text { de autocuidado }\end{array}$ \\
\hline $\begin{array}{l}\text { Silva et al., Revista } \\
\text { Brasileira de Enfer- } \\
\text { magem Brasil, } 2014\end{array}$ & $\begin{array}{l}\text { Utilização de experiências } \\
\text { musicais como terapia } \\
\text { para sintomas de náusea e } \\
\text { vômito em quimioterapia }\end{array}$ & $\begin{array}{l}\text { Amostragem por con- } \\
\text { veniência composta } \\
\text { por } 13 \text { pacientes } \\
\text { admitidos no ambu- } \\
\text { latório de quimiote- } \\
\text { rapia com moderado } \\
\text { e auto potencial } \\
\text { emetogênico }\end{array}$ & $\begin{array}{l}\text { Musicote- } \\
\text { rapia }\end{array}$ & $\begin{array}{l}\text { Utilizar tecnologias } \\
\text { alternativas na dimi- } \\
\text { nuição do potencial } \\
\text { emetogênico dos } \\
\text { pacientes submetidos } \\
\text { a quimioterapia }\end{array}$ & $\begin{array}{c}\text { A música influenciou } \\
\text { minimamente nos sinais } \\
\text { vitais, no entanto foi eficaz } \\
\text { na diminuição de náuseas e } \\
\text { vômitos }\end{array}$ \\
\hline
\end{tabular}

As tecnologias leve-duras podem ser compreendidas como a utilização de conhecimentos por meio de saberes estruturados (teorias, modelos de cuidado, cuidado de enfermagem), e não precisam de um recurso de alta tecnologia para sua realização. Dentre elas o Processo de Enfermagem tem reafirmado o papel do enfermeiro tornando-o mais autônomo, científico, visível e emponderado do seu papel asistencial junto ao paciente ancológico em uso de quimioterapia ${ }^{(20)}$.

Nesse sentido, autores ${ }^{(21)}$ utilizaram o Processo de Enfermagem junto a estudantes de enfermagem e pacientes em tratamento quimioterápico, este por sua vez favoreceu o raciocínio clinico dos estudantes, aumentando a capacidade de pensar criticamente, raciocinar, usar a informação para aquisição de conhecimento, compreensão do mundo e tomada de decisão apropriadas à um indivíduo em tratamento quimioterápico. Outros aspectos positivos dessa tecnologia foram descritos por autores ${ }^{(22)}$ ao citarem que o Processo de Enfermagem (PE) consiste em um instrumento metodológico empregado para favorecer o cuidado, além de organizar as condições necessárias para a sua ocorrência.

Nessa mesma perspectiva supracitada, estudo(22) elaborou e implementou um instrumento de coleta de dados com foco no Processo de enfermagem, obtendo como resultado o aumento da visibilidade do papel do enfermeiro e também da qualidade da assistencia prestada ao paciente em uso de quimioterapia, o estudo contou com 12 enfermeiros e 20 pacientes. Semelhantemente, autores ${ }^{(23)}$ elaboraram um instrumento de coleta de dados para operacionalizar o Processo de Enfermagem em 20 pacientes com câncer de pulmão em quimioterapia ambulatorial e concluiram que após o teste de confiabilidade, o instrumento mostrou-se eficaz para conhecer o paciente, bem como o tipo de tratamento a ser adotado, além de requisitos básicos para a realização do autocuidado.

Assim, as tecnologias se materializam também em produtos educativos, lúdicos e esclarecedores, como foi observado na pesquisa(24) ao construir e validar uma cartilha informativa para 23 acompanhantes de pacientes pediátricos em uso de quimioterapia, trazendo esclarecendos aos acompanhantes e aumentando a segurança das informações prestadas a este tipo de públicopúblico específico.

A música também foi utilizada como tecnologia leve dura através dos estudos $^{(25,26)}$. O primeiro utilizou a músicoterapia para analisar as percepções de familiares de pessoas portadoras de câncer, já o segundo utilizou a música como terapia para sintomas de náusea e vômito para pacientes em quimioterapia. Em ambos os casos a musicoterapia mostrou-se benéfica ao promover a abertura do ser para o diálogo e o vínculo entre enfermeiro, cliente e família, pormovendo também a diminuição dos sintomas de náuseas e vômitos e consequentemente aumentando a qualidade de vida dos pacientes.

Autores $^{(27)}$, ao estudarem a qualidade de vida (QV) de 48 pacientes com câncer colorretal em uso de quimioterapia ambulatorial, perceberam 
que a QV é resultado da combinação de fatores subjetivos (como o grau de satisfação geral de um individuo com a própria vida) e de fatores objetivos, como o bem estar material, boas relações familiares, disposição para tratamento do câncer, dentre outros fatores como tranqüilidade, confiança, segurança e bem estar. Para autores ${ }^{(28)}$, cabe ao enfermeiro avaliar o paciente sistematicamente afim de desenvolver um cuidado eficiente, autêntico e de qualidade. Assim, é necessário considerar em suas ações aspectos essenciais à relação humano-humano que caracterizam as tecnologias leves, como: a conversa, o saber ouvir, o toque, o compartilhamento de idéias, a demonstração de preocupação e a expressão de afeto, estar atento aos desejos e reivindicações, e, ainda, outros aspectos que são valorizados na visão holística do cuidado.

Dentre as tecnologias duras encontradas na pesquisa realizada se destacaram o dispositivo para infusão continua sofware computacional, brinquedo terapêutico e vídeo educativo. Para autores ${ }^{(20)}$, a tecnologia dura é exemplificada pelo uso de alta tecnologia, como bombas de infusão, ventiladores mecânicos e demais maquinários que demandam grande tecnologia. Além disso, também se classificam como tecnologia dura os softwares e vídeos, conforme evidenciado na Tabela 4.

As tecnologias duras envolvem produtos tecnológicos e são largamente utilizados pelos profissionais de enfermagem em serviços de terapia antineoplásica ${ }^{(3)}$. Autores ${ }^{(29)}$, em pesquisa realizada com 130 estudantes de enfermagem, utilizaram um vídeo educativo sobre a higiene bucal e perceberam que houve melhora na prática de higiene oral dos pacientes em uso de quimioterapia, diminuindo a proliferação de microrganismo e aumentando a qualidade de vida alimentar, proporcionando ao mesmo tempo o aumento da capacidade de cognição e mudança de comportamento dos estudantes frente aos resultados da pesquisa. Autores ${ }^{(30)}$, ao utilizarem vídeo educativo para prevenção e detecção precoce do câncer de mama, concluíram que essa tecnologia foi muito útil ao proporcionar motivação aos pacientes, aumentando a autoesti-

Tabela 4. Análise dos Artigos selecionados segundo classificação das Tecnologias - "Dura". Vitória, ES, Brasil, 2017

\begin{tabular}{|c|c|c|c|c|c|}
\hline Referências & Nome do Artigo & $\begin{array}{c}\text { Característica da } \\
\text { Amostra/População }\end{array}$ & $\begin{array}{l}\text { Tecnologia } \\
\text { estudada }\end{array}$ & Papel da Enfermagem & $\begin{array}{c}\text { Resultados / Expecta- } \\
\text { tivas }\end{array}$ \\
\hline $\begin{array}{l}\text { Siqueira et al. Revista } \\
\text { da Rede de Enferma- } \\
\text { gem do Nordeste Brasil, } \\
2013\end{array}$ & $\begin{array}{c}\text { Utilização de } \\
\text { dispositivo para } \\
\text { infusão contínua } \\
\text { de quimioterápico } \\
\text { na percepção do } \\
\text { paciente onco- } \\
\text { lógico }\end{array}$ & $\begin{array}{l}\text { Avaliou a percepção de } \\
8 \text { pacientes em uso de } \\
\text { dispositivo para infusão } \\
\text { continua. }\end{array}$ & $\begin{array}{c}\text { Dispositivo } \\
\text { para Infusão } \\
\text { Contínua }\end{array}$ & $\begin{array}{l}\text { Identificar nos pacientes } \\
\text { a necessidade do uso } \\
\text { do dispositivo para } \\
\text { infusão continua. }\end{array}$ & $\begin{array}{l}\text { Diminuição das hospita- } \\
\text { lizações, desvendando o } \\
\text { desconhecido e auxiliando } \\
\text { no desempenho das } \\
\text { atividades }\end{array}$ \\
\hline
\end{tabular}

Avaliação de orientações gera-

Lopes e Shmeil, Revista Gaúcha de Enfermagem Brasil, 2016 das por sistema computacional a acompanhantes de pacientes pediátricos submetidos à quimioterapia

Uso do brinquedo

Artilheiro, Almeida e Chacon, ACTA paulista de Enfermagem Brasil, 2011

terapêutico no preparo de crianças pré escolares para quimioterapia ambulatorial

Efeito de vídeo educativo no conhecimento do aluno sobre higiene bucal de pacientes em quimioterapia
8 enfermeiros e 50 acompanhantes de paciente pediátricos em uso de terapia antineoplásica
Software computacional

Realizar orientações de enfermagem utilizando a tecnologia de Software computacional
As orientações inseridas em um sistema possibilitaram a tomada de decisão e o julgamento clínico do enfermeiro
Stina, Zamarioli e CarvaIho, Escola Anna Nery Revista de Enfermagem Brasil, 2015

\section{Pesquisa realizada com 30 crianças com idade de 3 a 6 anos submetidas a quimioterapia}

130 estudantes de enfermagem da escola de Enfermagem de Ribeirão Preto
Video educativo

Brinquedo Terapêutico
Descrever o uso do brinquedo terapêutico identificando as reações das crianças, correlacionando-as com o uso da quimioterapia

Verificar a eficácia do vídeo educativo no conhecimento cognitivo e procedimental de alunos de enfermagem.
As crianças se mostraram mais colaborativas, interagindo com os adultos e cooperando com os procedimentos

0 vídeo contribuiu para melhorar cognitivo e procedimental dos alunos, proporcionou mudança de comportamento imediata, sendo uma estratégia bem aceita. 
ma da mulher e emponderando-a sobre a sua fenimilidade.

Já o uso de brinquedos terapêuticos mostrou-se relevante no preparo de crianças pré escolares para quimioterapia ambulatorial, diminuindo o medo do desconhecido e auxiliando na adesão ao tratamento ${ }^{(31)}$. Outro estudo mostrou que após utilizar a mesma tecnologia supracitada houve claramente uma diminuição e superação da ansiedade por parte das crianças, além da diminuição dos efeitos colaterais gerados pelo tratamento antineoplásico ${ }^{(32)}$.

Já o uso de software, também evidenciado na literatura pesquisada, foi utilizado para orientação de acompanhantes e pacientes pediátricos submetidos à quimioterapia, trazendo benefícios cognitivos e auento da adesão ao tratamento por parte dos pacientes em uso de quimioterapia ${ }^{(33)}$, tal achado corrobora com os resultados da pesquisa $^{(17)}$ que, ao desenvolver e utilizar um software para o ensino dos sinais vitais identificou que a tecnologia contribuiu para o aprendizado in- terativo, atrativo e lúdico, destacando a usabilidade de forma atemporal e sem limites geográficos.

O uso de dispositivos para infusão continua também foram encontrados na pesquisa (tecnologia dura). Autores $^{(34)}$ identificaram que esses dispositivos favorecem os pacientes em tratamento quimioterápico, contribuindo para o bem estar e aumentando as possibilidades de ir para casa, poder dormir na sua cama, ficar ao lado dos familiares o que é bastante positivo durante o tratamento do paciente oncológico.

\section{CONCLUSÃO}

A partir dos estudos analisados foi possível compreender o contexto em que estão inseridos os profissionais de enfermagem no uso de novas tecnologias no tratamento antineoplásico. Percebe-se que estas tecnologias são bem vindas pelos profissionais, sejam elas duras, leve-duras ou leves, à medida que são empregadas corretamente se tornam benéficas e aplicáveis aos pacientes submetidos à terapia antionaplásica.

As tecnologias utilizadas pelos profissionais de enfermagem em uso de quimioterapia foram vídeo educativo, brinquedo terapêutico, software, dispositivo para infusão contínua, processo de enfermagem, músicoterapia, comunicação, acolhimento e relação interpessoal. Foi possível identificar a relevância atribuída aos novos tratamentos, visto que a enfermagem presta aos seus clientes uma assistência humanizada e diferenciada. Contudo, a partir dos resultados expostos nesta pesquisa, verifica-se que o surgimento de novas tecnologias para o tratamento quimioterápico é norteado de benefícios aos pacientes portadores da doença e a toda equipe multidisciplinar, principalmente o enfermeiro.

Ressalta-se que novos estudos devem ser realizados a fim de identificar o uso de novas tecnologias em âmbito internacional, pois o presente estudo limitou-se a analisar as tecnologias utilizadas por enfermeiros nos serviços de terapia antineoplásica brasileiro.

\section{Referências}

1. Lorenzetti J, Trindade LL, Pires DEP, Ramos FRS. Tecnologia, inovação tecnológica e saúde: uma reflexão necessária. Texto Contexto Enfermagem [Internet]. 2012 [acesso em $18 \mathrm{dez}$ 2017]; 2(24):232239. Disponível em: http://www.scielo.br/pdf/tce

/v21n2/a23v21n2.pdf.

2. Frota NM, Barros LM, Araújo TM, Lopes MVO, Almeida PC, Caetano JA. Validação de hipermídia educativa sobre punção venosa periférica. Texto Contexto Enfermagem [Internet]. 2015 [acesso em 27 nov 2017]; 2(24):353-361. Disponível em: http://repositorio.ufc. br/bitstream/riufc/16422/1/2015_art_nmfrota.pdf.

3. Prado C, Perez HHC, Leite MMJ. Tecnologia da informação e comunicação em enfermagem. 1. ed. São Paulo: Atheneu; 2011.

4. Mehry EE. Saúde: cartografia do trabalho vivo em ato. 2. ed. São Paulo: Hucitec; 2002.

5. Nietsche E. Tecnologias educacionais, assistenciais e gerenciais: uma reflexão a partir da concepção dos docentes de enfermagem. Revista Latino-Americana Enfermagem [Internet]. 2015 [acesso em 22 jan 2018]; 13(3):344-353. Disponível em: http://www.revistas. usp.br/rlae/article/view/2091/2177.

6. Bonassa EMA, Gato MIR. Terapêutica oncológica para enfermeiros e farmacêuticos. 4. ed. São Paulo: Atheneu; 2012.

7. Ministério da Saúde (BR). Ações de enfermagem para o controle do câncer: uma proposta de integração ensino-serviço. 3. ed. Rio de
Janeiro: INCA, 2008.

8. Conselho Federal de Enfermagem (BR). Resolução n. ${ }^{\circ} 358$, de 15 de outubro de 2009. Brasília 15 out, 2009 [acesso em 23 out 2017]. Disponível em: http://www.cofen.gov.br/ resolucao-cofen3582009_4384.html.

9. Fonseca LMM, Goes FSN, Ferrecini GM, Leite AM, Mello DF, Scochi CGS. Inovação tecnológica no ensino da semiotécnica e semiologia em enfermagem neonatal: desenvolvimento à utilização de um software educacional. Texto Contexto Enfermagem [Internet]. 2009 [acesso em 15 jan 2018]; 3(18):549-558. Disponível em: http:// www.scielo.br/pdf/tce/v18n3/a19v18n3.pdf.

10. Souza MT, Silva MD, Carvalho R. Revisão integrativa: o que é e como fazer. Einstein [Internet]. 2010 [acesso em 02 jan 2018]; 1(8):102-106, 2010. Disponível em: http://www.scielo.br/pdf/eins/ v8n1/pt_1679-4508-eins-8-1-0102.pdf.

11. Ursi ES, Gavão CM. Prevenção de lesões de pele no perioperatório: revisão integrativa da literatura. Revista Latino-Americana Enfermagem [Internet]. 2006 [acesso em 20 dez 2017]; 14(1):124-131. Disponivel em: http://www.scielo.br/pdf/rlae/v14n1/v14n1a17.pdf. 12. Crozeta K, Stocco JGD, Labronici LM, Méier MJ. Interface entre a ética e um conceito de tecnologia em enfermagem. Acta Paulista Enfermagem [Internet]. 2010 [acesso em 16 nov 2017]; 2(23):239243. Disponível em: http://www2.unifesp.br/acta/pdf/v23/n2/ 
v23n2a14.pdf.

13. Silva RCL, Porto IS, Figueiredo NMA. Reflexões acerca da assistência de enfermagem e o discurso de humanização em terapia intensiva. Escola Anna Nery Enfermagem [Internet]. 2008 [acesso em 10 jan 2018]; 1(12):156-159. Disponível em: http://www.scielo. br/pdf/ean/v12n1/v12n1a24.pdf.

14. Peterson AA, Carvalho EC. Comunicação terapêutica na enfermagem: dificuldades para o cuidar de idosos com câncer. Revista Brasileira de Enfermagem [Internet]. 2011 [acesso em 05 jan 2018]; 4(64):692-697. Disponível em: http://www.scielo.br/pdf/reben/ v64n4/a10v64n4.pdf.

15. Marques DLL, Laranja COL, Silva MCM. Interação entre família e equipe de enfermagem: repercussões na terapêutica do paciente oncológico. Revista de Enfermagem UFPE On Line [Internet]. 2014 [acesso em 12 nov 2017]; 8(8):2811-2815. Disponível em: https:// periodicos.ufpe.br/revistas/revistaenfermagem/article/viewFile/9988/1340.

16. Araújo MMT, Silva MJP. A comunicação com o paciente em cuidados paliativos: valorizando a alegria e o otimismo. Rev Esc Enferm USP [Internet]. 2007 [acesso em 13 jan 2018]; 4(41):668-674. Disponível em: http://www.scielo.br/pdf/reeusp/v41n4/17.pdf.

17. Pontes $A C$, Leitão IMTA, Ramos IC. Comunicação terapêutica em enfermagem: instrumento essencial do cuidado. Revista Brasileira de Enfermagem [Internet]. 2008 [acesso em 14 dez 2017]; 3(61):312-318, 2008. Disponível em: http://www.scielo.br/pdf/reben/v61n3/a06v61n3.pdf.

18. Peres MFP, Arantes ACLQ, Lessa OS, Caous CA. A importância da integração da espiritualidade e da religiosidade no manejo da dor e dos cuidados paliativos. Revista de Psiquiatria Clínica [Internet]. 2007 [acesso em 03 jan 2018]; 1(34):82-87. Acesso em: http://www. scielo.br/pdf/rpc/v34s1/a11v34s1.pdf.

19. Souza VM, Frizzo HCF, Paiva MHP, Bousso RS, Santos ASS. Espiritualidade, religiosidade e crenças pessoais de adolescentes com câncer. Revista Brasileira de Enfermagem [Internet]. 2015 [acesso em 15 dez 2017]; 5(68):791-796. Disponível em: http://www.scielo. br/pdf/reben/v68n5/0034-7167-reben-68-05-0791.pdf.

20. Sabino LMM, Brasil DRM, Caetano DRM, Santos MCL, Alves MDS. Uso de tecnologia leve-dura nas práticas de enfermagem: análise de conceito. Chia [Internet]. 2016 [acesso em 14 nov 2017]; 16(2):230-239. Disponível em: http://www.scielo.org.co/pdf/aqui/ v16n2/v16n2a10.pdf.

21. Anjos ACY, Megnabosco P, Borges DO, Campos CS. Sistematização da Assistência de Enfermagem ao paciente em tratamento quimioterápico antineoplásico: relato de experiência. Revista em Extensão [Internet]. 2011 [acesso em $14 \mathrm{dez}$ 2017]; 10(1):107-112. Disponível em: http://www.seer.ufu.br/index.php/revextensao/article/view/20628/10985.

22. Santos NSN, veiga $P$, Andrade R. Importância da anamnese e do exame físico para o cuidado do enfermeiro. Revista Brasileira de Enfermagem [Internet]. 2011 [acesso em 12 dez 2017]; 64(2):355-358. Disponível em: http://www.scielo.br/pdf/reben/v64n2/a21v64n2. pdf.

23. Salvadori AM, Lamas JLT, Zanon C. Desenvolvimento de Instrumento de coleta de dados de enfermagem para pacientes com câncer de pulmão em quimioterapia ambulatorial. Escola Anna Nery Enfermagem [Internet]. 2008 [acesso em 18 jan 2017]; 12(1):130135. Disponível em: http://www.scielo.br/pdf/ean/v12n1/v12n1a20. pdf.

24. Salles PS, Castro RCBR. Validação de material informativo a pacientes em tratamento quimioterápico e aos seus familiares. Revista Escola Paulista Enfermagem [Internet]. 2010 [acesso em $10 \mathrm{dez}$
2017]; 1(44):182-189. Disponível em:< http://www.scielo.br/pdf/ reeusp/v44n1/a26v44n1.pdf.

25. Silva VA, Marcon SS, Sales CA. Percepções de familiares de pessoas portadoras de câncer sobre encontros musicais durante o tratamento antineoplásico. Revista Brasileira de Enfermagem [Internet]. 2014 [acesso em 12 jan 2018]; 3(67):408-414. Disponível em: http://www.scielo.br/pdf/reben/v67n3/0034-7167-reben-67-03-0408.pdf.

26. Silva GJ, Fonseca MS, Rodrigues AB, Oliveira PP, BRASIL DRM, Moreira MMC. Utilização de experiências musicais como terapia para sintomas de náusea e vômito em quimioterapia. Revista Brasileira de Enfermagem [Internet]. 2014 [acesso em 20 dez 2017]; 4(67):630-636. Disponivel em: http://www.scielo.br/pdf/reben/ v67n4/0034-7167-reben-67-04-0630.pdf.

27. Chaves PL, Gorini MIPC. Qualidade de vida do paciente com câncer colorretal em quimioterapia ambulatorial. Revista Gaúcha de Enfermagem [Internet]. 2011 [acesso em 12 nov 2017]; 4(32):276-275. Disponivel em: http://www.scielo.br/pdf/rgenf/ v32n4/v32n4a18.Pdf.

28. Machado SM, Sawada NO. Avaliação da qualidade de vida de pacientes oncológicos em tratamento quimioterápico adjuvante. Texto Contexto Enfermagem [Internet]. 2008 [acesso em 14 jan 2018]; 4(17):750-757. Disponível em: http://www.scielo.br/pdf/tce/ v17n4/17.pdf.

29. Stina APN, Zamarioli CM, Carvalho EC. Efeito de vídeo educativo no conhecimento do aluno sobre higiene bucal de pacientes em quimioterapia. Escola Anna Nery Enfermagem [Internet]. 2015 [acesso em 19 jan 2018]; .2(19):220-225. Disponível em: http://www.scielo. br/pdf/ean/v19n2/1414-8145-ean-19-02-0220.pdf>.

30. Moreira CB, Bernardo EBR, Catunda HLO, Aquino OS, Santos $\mathrm{MCL}$, Fernandes AFC. Construção de um Vídeo Educativo sobre Detecção Precoce do Câncer de Mama. Revista Brasileira de Cancerologia [Internet]. 2013 [acesso em 18 dez 2018]; 3(59):401-407, 2013. Disponível em: http://www1.inca.gov.br/rbc/n_59/v03/pdf/10-artigo-construcao-video-educativo-sobre-deteccao-precoce-cancer-mama.pdf.

31. Artilheiro APS, Almeida FAA, Chacon JMF. Uso do brinquedo terapêutico no preparo de crianças préescolares para quimioterapia ambulatorial. Acta Paulista Enfermagem [Internet]. 2011 [acesso em 21 dez 2017]; 5(24):611-616. Disponível em: http://www.scielo. $\mathrm{br} / \mathrm{pdf} / \mathrm{ape} / \mathrm{v} 24 \mathrm{n} 5 / 03 \mathrm{v} 24 \mathrm{n} 5$. pdf.

32. Schenke IC, Garcia JM, Batista MSK, Schivinski CIS, Silva MEM. Brinquedo terapêutico como coadjuvante ao tratamento fisioterapêutico de crianças com afecções respiratórias. Revista de Psicologia Teoria e Prática [Internet]. 2013 [acesso em 19 nov 2017]; 1(15):130-144. Disponível em: http://pepsic.bvsalud.org/pdf/ptp/ v15n1/11.pdf.

33. Lopes VJ, Shmell MAH. Avaliação de orientações geradas por sistema computacional a acompanhantes de pacientes pediátricos submetidos à quimioterapia. Revista Gaúcha de Enfermagem [Internet]. 2016 [acesso em 22 dez 2017]; 2(37):01-09. Disponível em: http://www.scielo.br/pdf/rgenf/v37nspe/0102-6933-rgenf-1983 14472016esp67407pdf.

34. Siqueira JFS, Silva DMAS, Oliveira FJG, Campos FA, Camurça MNS, Caetano JA. Utilização de dispositivo para infusão contínua de quimioterápico na percepção do paciente oncológico. Revista RENE [Internet]. 2013 [acesso em 12 jan 2018]; 6(14):12171223. Disponível em: http://www.repositorio.ufc.br/bitstream/riufc/11357/1/2013_art_jfsiqueira.pdf. 